NOTULES HYDRAULIQUES

HYDRAULIC BRIEFS

\title{
Remarques
}

\section{sur la similitude des galeries en charge}

\section{Notes on the similitude \\ of full-flowing tunnels}

English synopsis, p. 107.

L'étude hydraulique d'une vidange de fond ou d'une galerie de dérivation provisoire conduit très souvent à exécuter un modèle réduit qui, seul, permet de résoudre certains problemes.

Nous adopterons les notations suivantes:

I. : Iongueur de la galerie.

$\mathrm{H}$ : charge amont.

C : coefficient de ChÉzy.

$R_{n}$ : rayon hydraulique.

Q : débit.

$\mathrm{S}$ : section.

s : coefficient de débit.

Les notations relatives au modèle réduit seront caractérisées par «prime».

La conception de la maquette et l'interprétation des résultats ne peuvent se faire sans certaines précautions que cette note a pour objet d'exposer.

\section{Hypotheses}

- La galerie est en charge au débit maximum (1).

(1) Nous étudierons dans un autre article le cas de la surface libre.
-.- Les coefficients $C^{\prime}$ et $C^{\prime}$ de CHEzy sont constants (ceci est d'ailleurs unc conséquence do la première hypothèse).

Les coefficients de débit a et $\alpha^{\prime}$ sont identiques sur le modèle et dans la réalité.

Rappelons l'équation génćrale:

$$
\mathrm{Q}=x \sqrt{2 g} \mathrm{~S} \sqrt{\mathrm{H}-\frac{\mathrm{V}^{2} \mathrm{~L}}{\mathrm{C}^{2} \mathrm{R}_{h}}}
$$

la perte de charge répartie dans la galerie est représentée par :

$$
\frac{V^{2} L}{C^{2} R_{h}}=\frac{Q^{2} L}{C^{2} S^{2} R_{h}}
$$

La résolution en $Q$ de l'équation (1) donne :

$$
\mathrm{Q}=\alpha \mathrm{S} \sqrt{2 g} \sqrt{\mathrm{H}} \frac{1}{\sqrt{1+\frac{2 g \alpha^{2} \mathrm{~L}}{\mathrm{C}^{2} \mathrm{R}_{\pi}}}}
$$

La perte de charge peut dono s'écrire :

$$
\text { p.d.c. }=\frac{2 g \alpha^{2} \mathrm{~L}}{\mathrm{C}^{2} \mathrm{R}_{h}+2 g \alpha^{2} \mathrm{~L}} \mathrm{H}
$$

Soit $\lambda$ l'échelle adoptée pour le modèle. Nous avons les relations suivantes:

$$
\begin{aligned}
\mathrm{R}_{h} & =\lambda \mathrm{R}_{h}^{\prime} \\
\alpha & =\alpha^{\prime} \\
\mathrm{S} & =\lambda^{2} \mathrm{~S}^{\prime}
\end{aligned}
$$


Nous devons, de plus, avoir similitude entre les pertes de charge, ce qui s'écrit :

$$
\mathrm{H}\left[\frac{\mathrm{C}^{\prime 2} \mathrm{R}_{h}^{\prime}}{2 g \alpha^{2} \mathrm{~L}^{\prime}}+1\right]=\lambda \mathrm{H}^{\prime}\left[\frac{\mathrm{C}^{2} \mathrm{R}_{h}}{2 g \alpha^{2} \mathrm{I}}\right]+1
$$

Les pertes de charge reparties sur le modele et dans la réalité dépendent essentiellement des coefficients de rugosité $C^{\prime}$ et $C$.

-- pour le modèle, il est préférable d'employer la formule de Bazrn dans laquelle te coefficient sera de l'ordre de 0,06 ;

- pour la réalité, C est également une donnée qui dépend du mode d'exécution de la gaIerie.

Deux cas peuvent se présenter à l'expérimenLateur :

1) Il faut obligatoirement le débit en similitude sur le modèle, pour des études d'afronillement ou de ressant aval par exemple;

2) La similitude non distordue des formes est nécessaire.

Etudions les deux cas :

On peut jouer sur la longueur de la galerie, et soit $l$ la longueur qui doit remplacer $L^{\prime}$ sur le modele, l'équation (5) donne alors:

$$
l=\frac{\mathrm{L}}{\lambda}\left(\frac{\mathrm{C}^{\prime}}{\mathrm{C}}\right)^{2}
$$

-... Soit $Q$ le débit mesuré sur le modèle non distordu, calculons le débit q auquel il correspond réellement.

Par analogic avec l'épuation (3), nous écrirons :

$$
\mathrm{Q}^{\prime}=\alpha \mathrm{S}^{\prime} \sqrt{2 g} \cdot \sqrt{\mathrm{H}^{\prime}} \frac{1}{\sqrt{1+\frac{2 g \alpha^{2} \mathrm{~L}^{\prime}}{\mathrm{C}^{\prime \prime}-\mathrm{R}^{\prime}}}}
$$

en résolvant avec l'équation (5), on obtient :

$$
q=Q^{\prime} \sqrt{\frac{\mathrm{C}^{\prime 2} \mathrm{R}^{\prime}+2 g \alpha^{\prime 2} \mathrm{~L}^{\prime}}{\mathrm{C}^{\prime \prime 2} \mathrm{R}^{\prime}+2 g \alpha^{2}\left(\frac{\mathrm{C}^{\prime}}{\mathrm{C}}\right)^{2} \mathrm{~L}^{\prime}}}
$$

et

$$
Q=q^{25 / 2}
$$

\section{J. SOULET,}

Ingénieur au Laboratoire. Dauphinois d'Hydraulique

(Neyrpic - Grenoble)

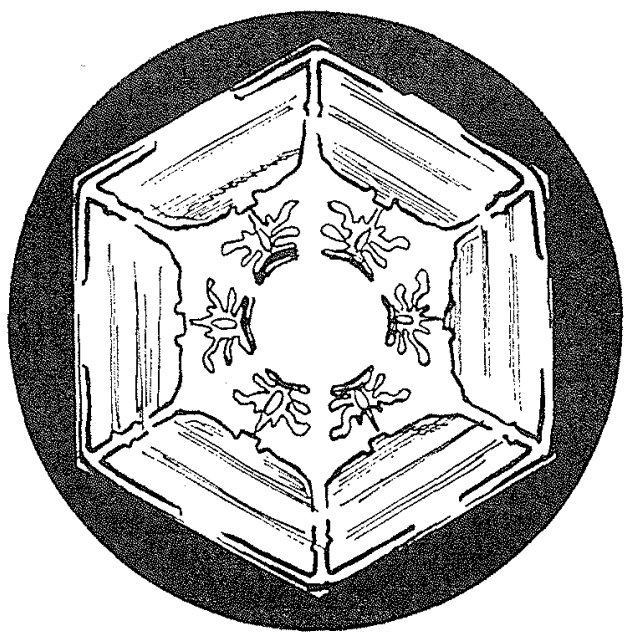

